\title{
Amla Enhances Mitochondrial Spare Respiratory Capacity by Increasing Mitochondrial Biogenesis and Antioxidant Systems in a Murine Skeletal Muscle Cell Line
}

\author{
Hirotaka Yamamoto, ${ }^{1,2}$ Katsutaro Morino, ${ }^{2}$ Lemecha Mengistu, ${ }^{2}$ Taishi Ishibashi, ${ }^{1}$ \\ Kohei Kiriyama, ${ }^{1}$ Takao Ikami, ${ }^{1}$ and Hiroshi Maegawa ${ }^{2}$ \\ ${ }^{1}$ Institute for Health Science, MIKI Corporation, 3-12-4, Naruohama, Nishinomiya, Hyogo 663-8142, Japan \\ ${ }^{2}$ Department of Medicine, Division of Endocrinology and Metabolism, Shiga University of Medical Science, Tsukinowa, Seta, \\ Otsu, Shiga 520-2192, Japan \\ Correspondence should be addressed to Katsutaro Morino; morino@belle.shiga-med.ac.jp
}

Received 26 December 2015; Revised 25 March 2016; Accepted 4 May 2016

Academic Editor: Thomas Kietzmann

Copyright (C) 2016 Hirotaka Yamamoto et al. This is an open access article distributed under the Creative Commons Attribution License, which permits unrestricted use, distribution, and reproduction in any medium, provided the original work is properly cited.

\begin{abstract}
Amla is one of the most important plants in Indian traditional medicine and has been shown to improve various age-related disorders while decreasing oxidative stress. Mitochondrial dysfunction is a proposed cause of aging through elevated oxidative stress. In this study, we investigated the effects of Amla on mitochondrial function in C2C12 myotubes, a murine skeletal muscle cell model with abundant mitochondria. Based on cell flux analysis, treatment with an extract of Amla fruit enhanced mitochondrial spare respiratory capacity, which enables cells to overcome various stresses. To further explore the mechanisms underlying these effects on mitochondrial function, we analyzed mitochondrial biogenesis and antioxidant systems, both proposed regulators of mitochondrial spare respiratory capacity. We found that Amla treatment stimulated both systems accompanied by AMPK and Nrf2 activation. Furthermore, we found that Amla treatment exhibited cytoprotective effects and lowered reactive oxygen species (ROS) levels in cells subjected to t-BHP-induced oxidative stress. These effects were accompanied by increased oxygen consumption, suggesting that Amla protected cells against oxidative stress by using enhanced spare respiratory capacity to produce more energy. Thus we identified protective effects of Amla, involving activation of mitochondrial function, which potentially explain its various effects on age-related disorders.
\end{abstract}

\section{Introduction}

Amla is one of the most important botanical materials in Indian traditional medicine, "Ayurveda." It has been used for many diseases including diabetes, osteoporosis, liver dysfunction, and anemia, not only in India but also in other countries $[1,2]$. Recently, the chemical composition of Amla was analyzed, showing relatively high levels of phenolic compounds [2]. A recent clinical study showed that Amla extract improved endothelial function in patients with type 2 diabetes mellitus [3]. Furthermore, its antioxidant [4], hepatoprotective [5], nephroprotective [6], hypolipidemic $[7,8]$, cardioprotective $[9,10]$, and antidiabetic effects [11] were demonstrated in in vivo animal models. One potential mechanism for these effects was reduction of oxidative stress, based on observations of decreased oxidative stress with Amla treatment. However, the detailed mechanisms have not yet been fully identified.

Accumulating evidence suggested a central role for mitochondria in aging through production of both energy and reactive oxygen species (ROS). Mitochondria can produce substantial energy through aerobic metabolism, though they also produce ROS as unwanted byproducts, causing serious damage to various cellular components. Because mitochondria are the major ROS producing organelle, they are also vulnerable to injury by ROS. Damaged mitochondria produce more ROS, leading to further mitochondrial damage [12]. These defective mitochondria exhibit impaired energy 
production and increased oxidative stress, both negatively impacting cellular function. Numerous reports implicated mitochondrial dysfunction in many pathologies or disorders related to the aging process $[13,14]$. Mitochondrial spare respiratory capacity is regarded as an important aspect of mitochondrial function and is defined as the difference between basal ATP production and its maximal activity. When cells are subjected to stress, energy demand increases, with more ATP required to maintain cellular functions. A cell with a larger spare respiratory capacity can produce more ATP and overcome more stress, including oxidative stress [15]. Therefore, we hypothesized that Amla could improve mitochondrial function, especially spare respiratory capacity, and exert positively effects on various disorders related to oxidative stress.

To explore the effects of Amla on mitochondrial function, we used a skeletal muscle cell known to contain abundant mitochondria. Using this model, we investigated the molecular mechanisms of Amla's beneficial effects.

\section{Materials and Methods}

2.1. Plant Materials. A commercial product of Amla fruit juice extract (SunAmla) was obtained from Taiyo Kagaku Co., Ltd. (Mie, Japan). It is a dried powder of water extract from fresh Amla fruit and was previously shown to contain $\sim 30 \%$ polyphenols and $2 \%$ vitamin C [6]. To confirm the equivalence of Amla used in this study, total polyphenol was analyzed by a colorimetric method using gallic acid as a standard [6], and vitamin C content was analyzed by highperformance liquid chromatography (HPLC) as previously reported [16]. Glucose and fructose in Amla were analyzed by HPLC as previously reported [17]. To prepare Amla extract stock solution, the extract was dissolved in distilled water at a concentration of $200 \mathrm{mg} / \mathrm{mL}$ and then sterilized using a polyvinylidene difluoride membrane filter (Merck Millipore, Darmstadt, Germany).

2.2. Reagents. Oligomycin, carbonyl cyanide-p-trifluoromethoxyphenylhydrazone (FCCP), rotenone, and antimycin A were obtained from Seahorse Bioscience (North Billerica, MA, USA) in XF Cell Mito Stress Test kit (\#103015100). Water soluble tetrazolium salts for the MTT assay were from Kishida Chemical Co., Ltd. (Osaka, Japan). $2^{\prime}, 7^{\prime}$-Dichlorofluorescein diacetate (D6883) and tert-butyl hydroperoxide (t-BHP; 458139) were obtained from SigmaAldrich (St. Louis, MO, USA).

2.3. Cell Culture. C2C12 myoblasts were obtained from the American Type Culture Collection (ATCC; Manassas, VA, USA) and grown in Dulbecco's modified Eagle's medium (DMEM) supplemented with $10 \%(\mathrm{v} / \mathrm{v})$ fetal bovine serum (FBS), 100 units $/ \mathrm{mL}$ penicillin, and $100 \mathrm{mg} / \mathrm{mL}$ streptomycin in a humidified atmosphere of $95 \%$ air and 5\% $\mathrm{CO}_{2}$ at $37^{\circ} \mathrm{C}$. At confluence, myoblasts were induced to differentiate in DMEM with $2 \%$ FBS, 100 units $/ \mathrm{mL}$ penicillin, and $100 \mathrm{mg} / \mathrm{mL}$ streptomycin. Differentiation medium was replaced every $48 \mathrm{~h}$. Human embryonic kidney 293
(HEK293) cells were obtained from the Japanese Collection of Research Bioresources cell bank (Osaka, Japan) and grown in Eagle's minimum essential medium with $10 \%$ (v/v) FBS, 100 units $/ \mathrm{mL}$ penicillin, and $100 \mathrm{mg} / \mathrm{mL}$ streptomycin in a humidified atmosphere of $95 \%$ air and $5 \% \mathrm{CO}_{2}$ at $37^{\circ} \mathrm{C}$. For Amla treatment, Amla stock solution was diluted with medium to final concentrations of $100 \mu \mathrm{g} / \mathrm{mL}$ and $200 \mu \mathrm{g} / \mathrm{mL}$.

2.4. Measurement of Mitochondrial Function. Mitochondrial function in $\mathrm{C} 2 \mathrm{C} 12$ myotubes was analyzed using the XFe24 flux analyzer with XF Cell Mito Stress Test kit according to manufacturer instructions (Seahorse Bioscience). Briefly, differentiated C2C12 myotubes were prepared on Seahorse 24 -well plates and treated with or without Amla $(100 \mu \mathrm{g} / \mathrm{mL}$ or $200 \mu \mathrm{g} / \mathrm{mL}$ ) for $48 \mathrm{~h}$. The culture medium was changed at least $40 \mathrm{~min}$ prior to the assay to unbuffered DMEM supplemented with $5 \mathrm{mM}$ glucose. The oxygen consumption ratio (OCR, $\mathrm{pmol} / \mathrm{min}$ ) was monitored in real time, with sequential treatments with oligomycin (ATP synthase inhibitor), FCCP (mitochondrial uncoupler), and rotenone/antimycin A (respiration inhibitor) to evaluate OCR from proton leak, maximum respiration capacity, and nonmitochondrial respiration, respectively. OCR was measured multiple times at $8 \mathrm{~min}$ intervals at each stage, and average values were determined. Nonmitochondrial respiration was subtracted from OCR at each stage to calculate the net OCR for Basal, Leak, and Max values. Mitochondrial respiratory spare capacity and ATP-transratio were calculated by the formula shown in Figure 1(a), left panel.

2.5. Nucleic Acid and Protein Isolation. Total DNA was isolated using a Qiamp DNA mini kit (Qiagen, Mississauga, $\mathrm{ON}$, Canada) according to manufacturer instructions. RNA and total protein were isolated using a PARIS kit (AM1921; Thermo Fisher Scientific, Waltham, MA, USA). Nuclear lysates were isolated using a nuclear/cytosol-fractionation kit (K266; Biovision, Inc., Milpitas, CA, USA) according to manufacturer protocol. Isolated protein samples were denatured by boiling in sodium dodecyl sulfate (SDS) sample buffer.

2.6. Western Blot. Proteins were separated by SDS-polyacrylamide gel electrophoresis and transferred onto polyvinylidene fluoride membranes. Immunoblotting was performed with primary antibodies against phosphorylated AMPK $\alpha$ (\#2531; Cell Signaling Technology, Danvers, MA, USA), AMPK $\alpha$ (\#2603; Cell Signaling Technology), Nrf2 (SC722; Santa Cruz Biotechnology, Dallas, TX, USA), and YY-1 (ab109237; Abcam, Tokyo, Japan), with horseradish peroxidase-conjugated secondary antibodies (GE Healthcare Japan, Tokyo, Japan). YY-1 was used as a nuclear-loading control. The intensity of protein bands was visualized using a chemiluminescence detection reagent (PerkinElmer, Inc., Waltham, MA, USA) and a WSE-6100 luminograph system (ATTO, Tokyo, Japan).

2.7. Quantitative PCR for Mitochondrial DNA ( $m t D N A)$ Content. mtDNA content was analyzed as previously described 


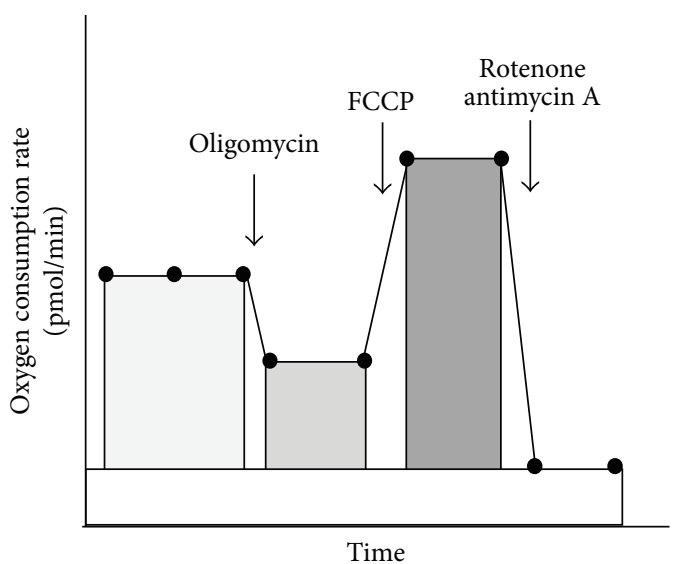

Mitochondrial spare capacity $=\mathrm{Max} /$ Base ATP-transratio $=($ Base - Leak $) /$ Base $\times 100$

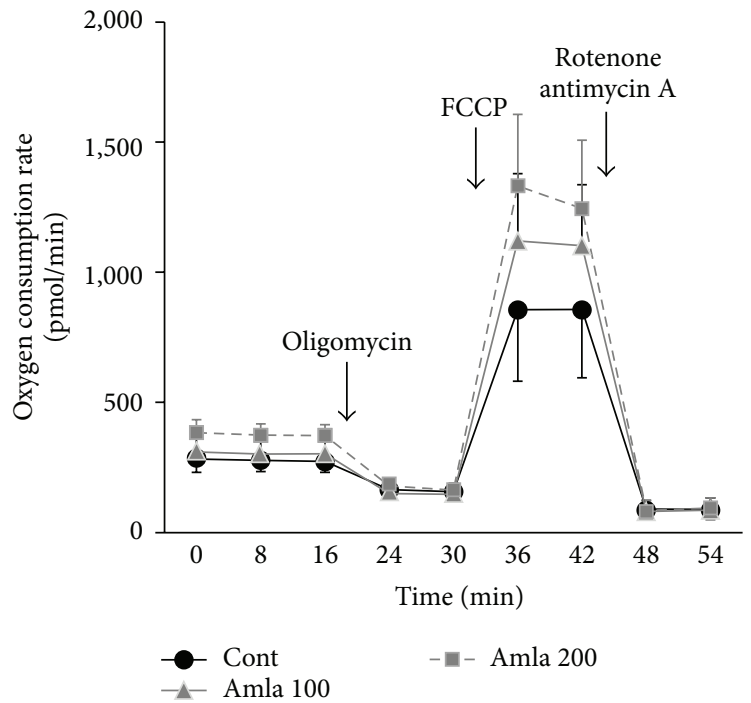

(a)

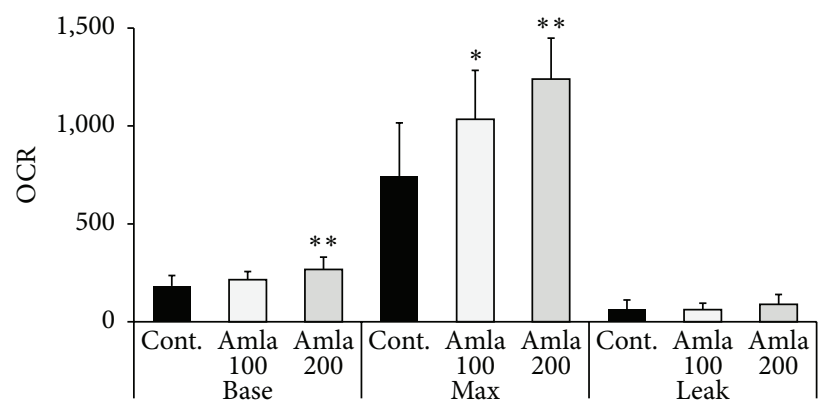

(b)

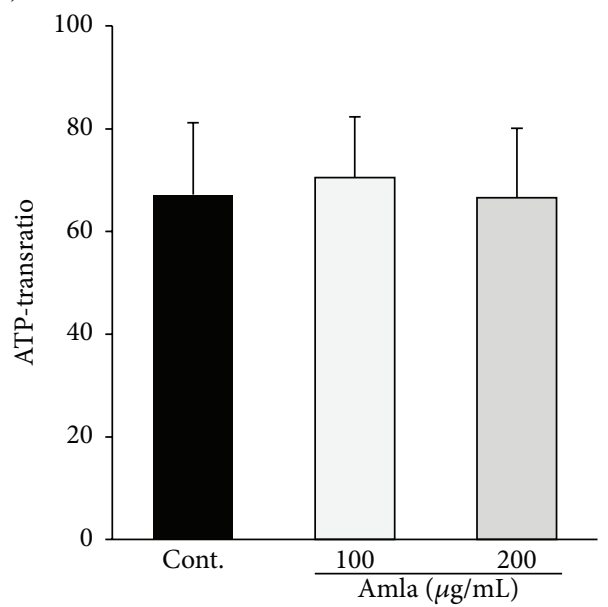

(d)

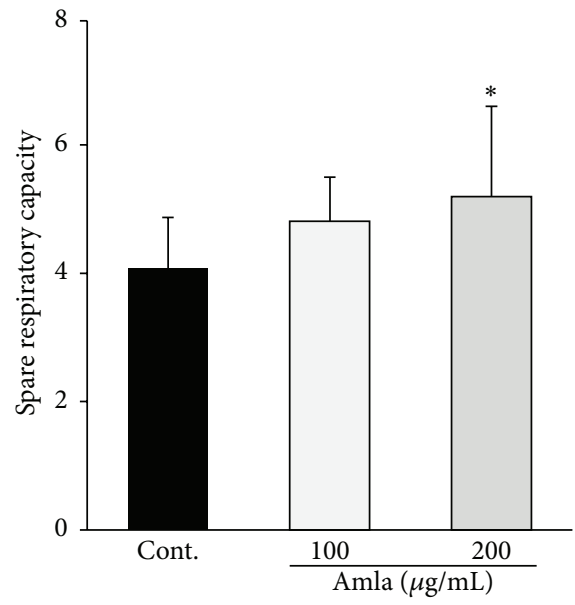

(c)

FIgURE 1: Amla treatment stimulated mitochondrial bioenergetic function. C2C12 myotubes were pretreated with two doses of Amla $(100 \mu \mathrm{g} / \mathrm{mL}$ or $200 \mu \mathrm{g} / \mathrm{mL})$ for $48 \mathrm{~h}$ and subjected to mitochondrial function analysis. ((a), left panel) Schematic figure analyzing mitochondrial function using an extracellular flux analyzer. After measuring basal OCR, oligomycin, FCCP, and rotenone/antimycin A were sequentially injected to measure OCR from proton leak, maximal respiratory capacity, and nonmitochondrial respiration, respectively. OCR from nonmitochondrial respiration was subtracted from OCR at each stage to calculate the net OCR for basal (Base), proton leak (Leak), and maximal respiratory capacity (Max) values. Mitochondrial spare respiratory capacity and ATP-transratio were calculated by the formula shown. ((a), right panel) OCR measurements over time ( $n=10$ or 11). (b) Basal, Max, and Leak OCRs represented as average values from multiple measurements. (c) Mitochondrial spare respiratory capacity was calculated from Max and Basal OCRs. (d) ATP-transratio was calculated from Basal and Leak OCRs. ${ }^{*} p<0.05 ;{ }^{* *} p<0.01$ as compared with control $(n=10$ or 11$)$. 
TABle 1: Primer sets used for RT-qPCR.

\begin{tabular}{|c|c|c|}
\hline Gene name & $\mathrm{F} / \mathrm{R}$ & Sequence $\left(5^{\prime}\right.$ to $\left.3^{\prime}\right)$ \\
\hline \multicolumn{3}{|l|}{ Internal standard } \\
\hline \multirow{2}{*}{$18 S$ rRNA } & $\mathrm{F}$ & TTCCGATAACGAACGAGACTCT \\
\hline & $\mathrm{R}$ & TGGCTGAACGCCACTTGTC \\
\hline \multicolumn{3}{|l|}{ Mitochondrial biogenesis } \\
\hline$P G C 1 \alpha$ & $\mathrm{F}$ & CCAAACCCACAGAAAACAGG \\
\hline PPAR-gamma coactivator 1-alpha & $\mathrm{R}$ & TGGGGTCATTTGGTGACTCT \\
\hline$N R F 1$ & $\mathrm{~F}$ & GAACTGCCAACCACAGTCAC \\
\hline Nuclear respiratory factor 1 & $\mathrm{R}$ & TCGTCTGGATGGTCATTTCA \\
\hline$m t T F A$ & $\mathrm{~F}$ & CCGAAGTGTTTTTCCAGCAT \\
\hline Mitochondrial transcriptional factor A & $\mathrm{R}$ & GGCTGCAATTTTCCTAACCA \\
\hline \multicolumn{3}{|l|}{ Antioxidant system } \\
\hline Nrf2 & $\mathrm{F}$ & GGGAGAAAACGACAGAAACC \\
\hline Nuclear factor erythroid 2 related factor 2 & $\mathrm{R}$ & TGGGAGAGTAAGGCTTTCCA \\
\hline HO-1 & $\mathrm{F}$ & TGACACCTGAGGTCAAGCAC \\
\hline Heme oxygenase-1 & $\mathrm{R}$ & TCCTCTGTCAGCATCACCTG \\
\hline NQO1 & $\mathrm{F}$ & AAACGTCTGGAAACCGTCTG \\
\hline Nadph dehydrogenase quinone 1 & $\mathrm{R}$ & TTCTGCTCCTCTTGAACTTCC \\
\hline Cat & $\mathrm{F}$ & GGACGCTCAGCTTTTCATTC \\
\hline Catalase & $\mathrm{R}$ & TTGTCCAGAAGAGCCTGGAT \\
\hline$G P x$ & $\mathrm{~F}$ & CTCATGACCGACCCCAAGTA \\
\hline Glutathione peroxidase & $\mathrm{R}$ & CССACCAGGAACTTCTCAAA \\
\hline$M n-S O D$ & $\mathrm{~F}$ & TCTGTGGGAGTCCAAGGTTC \\
\hline Manganese superoxide dismutase & $\mathrm{R}$ & TAAGGCCTGTTGTTCCTTGC \\
\hline $\mathrm{Cu}-\mathrm{SOD}$ & $\mathrm{F}$ & GAGACCTGGGCAATGTGACT \\
\hline Copper superoxide dismutase & $\mathrm{R}$ & TCATGGACCACCATTGTACG \\
\hline
\end{tabular}

[18]. DNA primers were designed to detect cytochrome oxidase 2 (COX2) and uncoupling protein 2 (UCP2) for mtDNA and nuclear DNA, respectively (COX2-F: $5^{\prime}$-TTTTCAGGCTTCACCCTAGATGA-3' COX2-R: $5^{\prime}$-GAAGAATGTTATGTTATGTTTACTCCTA-3' UCP2-F: 5'-GCGACCAGCCCATTGTAGA-3' UCP2-R: 5'-GCGTTCTGGGTACCATCCTAAC $-3^{\prime}$ ). The ratio of COX2 to UCP2 within each sample was used to calculate mtDNA content.

2.8. $m R N A$ Quantification. Complementary DNA was prepared using the PrimeScript2 1st strand cDNA synthesis kit (Takara, Otsu, Japan). RT-qPCR was performed in a StepOnePlus Real-Time PCR system (Thermo Fisher Scientific) using Fast SYBR Green Master Mix (Thermo Fisher Scientific) and primer pair sets described in Table 1. The $18 \mathrm{~S}$ rRNA was used as a housekeeping gene and served as an endogenous control.

2.9. Antioxidant Response Element (ARE) Luciferase Assay. The luciferase reporter plasmid (pGL4.26 luc2/minP/Hygro, E8441) and internal control plasmid (pRL-CMV, E2271) were from Promega (Madison, WI, USA). The DNA sequence for the ARE was obtained from the National Center for Biotechnology Information (GenBank: JQ858521.1), and a synthetic ARE polynucleotide was inserted into the HindIII site in multicloning site of pGL4.26 using the In-Fusion HD cloning kit (Takara). The ARE-loaded reporter and internal control vectors were cotransfected into HEK293 cells using lipofectamine-transfection reagent (Thermo Fisher Scientific). After a $24 \mathrm{~h}$ incubation, cells were treated with Amla $(200 \mu \mathrm{g} / \mathrm{mL})$ for another $48 \mathrm{~h}$, and luciferase activity was measured using the Dual-Luciferase Reporter Assay System (Promega).

2.10. MTT Measurements of Cell Viability under Oxidative Stress. C2C12 cells were seeded on 96-well plates and differentiated to myotubes as previously described in Section 2.3. To evaluate the cytoprotective effects of Amla on t-BHPinduced oxidative stress, cells were pretreated with or without Amla $(200 \mu \mathrm{g} / \mathrm{mL})$ for $48 \mathrm{~h}$, then with $\mathrm{t}-\mathrm{BHP}(250 \mu \mathrm{M}$ or $500 \mu \mathrm{M})$ for $6 \mathrm{~h}$. Cell viability was determined using a cellviability kit based on MTT reduction. Briefly, after treatment with t-BHP, cells were incubated with MTT assay reagents in culture medium for $20 \mathrm{~min}$, and absorbance at $495 \mathrm{~nm}$ was measured using an ARVO SX 1420 multilabel counter (PerkinElmer, Inc., Waltham, MA, USA). Cell viability was expressed as the percentage of values obtained with control cells not treated with t-BHP.

2.11. ROS Measurement. Intracellular ROS levels were analyzed using the fluorescent probe $2^{\prime}, 7^{\prime}$-dichlorofluorescein diacetate. Differentiated C2C12 myotubes in 96-well 
TABLE 2: Components of Amla extract.

\begin{tabular}{lcc}
\hline & \multicolumn{3}{c}{ Content, \% } \\
\hline Total polyphenols & 23.9 & SD \\
Vitamin C & 1.32 & 0.6 \\
Glucose & 3.5 & 0.2 \\
Fructose & 4.3 & 0.2 \\
\hline
\end{tabular}

Values are means of three replicate samples.

culture plates were pretreated with Amla $(200 \mu \mathrm{g} / \mathrm{mL})$ for $48 \mathrm{~h}$. Myotubes were incubated with $10 \mu \mathrm{M} 2^{\prime}, 7^{\prime}$ dichlorofluorescein diacetate for $30 \mathrm{~min}$ following $\mathrm{t}$ BHP treatment $(0 \mu \mathrm{M}, 250 \mu \mathrm{M}$, or $500 \mu \mathrm{M})$ for $2 \mathrm{~h}$. The fluorescence in cells, which was increased by ROS, was analyzed at $485 \mathrm{~nm} / 535 \mathrm{~nm}$ (excitation/emission) in an ARVO SX 1420 multilabel counter.

2.12. Oxygen Consumption Analysis in Response to Oxidative Stress. Amla $(200 \mu \mathrm{g} / \mathrm{mL})$ treated or untreated C2C12 myotubes in 24-well plates (Seahorse Bioscience) were prepared as described in Section 2.4. Basal OCR was measured three times at $8 \mathrm{~min}$ intervals prior to treatment. After administration of $\mathrm{t}$-BHP $(0 \mu \mathrm{M}, 250 \mu \mathrm{M}$, or $500 \mu \mathrm{M})$, OCR was then measured at $8 \mathrm{~min}$ intervals for $160 \mathrm{~min}$. Data are expressed as relative-OCR values normalized to basal OCR values.

2.13. Statistical Analysis. Results are means \pm standard deviation. Significance was assessed using Student's $t$-test and one-way analysis of variance. Differences among groups were determined using Tukey's multiple range test. A $p<0.05$ was considered significant.

\section{Results}

3.1. Amla Extract Components. The total polyphenol and vitamin C contents of Amla extract were $23.9 \%$ and $1.32 \%$, respectively (Table 2), and the extract contained low levels of glucose and fructose (3.5\% and $4.3 \%$, resp.). Because Amla extract was applied to cells at a concentration of $200 \mu \mathrm{g} / \mathrm{mL}$, extract-derived sugars $(\sim 0.8 \mathrm{mg} / \mathrm{dL}$ each $)$ were regarded as negligible.

3.2. Amla Treatment Enhanced Mitochondrial Spare Respiratory Capacity. To evaluate the effects of Amla treatment on mitochondrial function, we analyzed the OCR in $\mathrm{C} 2 \mathrm{C} 12$ myotubes with or without Amla pretreatment $(100 \mu \mathrm{g} / \mathrm{mL}$ or $200 \mu \mathrm{g} / \mathrm{mL}$ ). Nonmitochondrial respiration (Figure 1(a), right panel) and Leak (Figure 1(a), right panel, and Figure 1(b)) were unchanged by Amla treatment; however, Base and Max OCRs increased in a dose-dependent manner following Amla treatment (Figure 1(a), right panel, and Figure 1(b)). Mitochondrial spare respiratory capacity also increased in a dosedependent manner following Amla treatment, though the effects were not significant at lower Amla doses (Figure 1(c)). The ATP-transratio was unchanged by Amla treatment (Figure $1(\mathrm{~d})$ ). In order to clarify the mechanisms associated with
Amla treatment, we conducted further experiments using the higher dosage $(200 \mu \mathrm{g} / \mathrm{mL})$.

3.3. Amla Treatment Stimulated Mitochondrial Biogenesis by AMPK Activation. To elucidate how Amla treatment enhanced OCR and mitochondrial spare respiratory capacity, we evaluated its effects on mitochondrial biogenesis in $\mathrm{C} 2 \mathrm{C} 12$ myotubes. mtDNA copy number increased 1.5 -fold following Amla treatment (Figure 2(a)). To evaluate the molecular effects associated with Amla treatment, we analyzed activation of the AMPK $\alpha / \mathrm{PGCl} \alpha / \mathrm{NRF} 1 / \mathrm{mtTFA}$ pathway, a key regulatory pathway involved in mitochondrial biogenesis. In cells treated with Amla, AMPK $\alpha$-phosphorylation levels increased dramatically (Figure 2(b)), NRF1 and mtTFA mRNA expression increased significantly, and PGCl $\alpha$ mRNA exhibited trends indicating increased expression levels (Figure 2(c)).

3.4. Amla Treatment Stimulated Antioxidant Systems. In order to maintain mitochondrial function, ROS reduction and mitochondrial biogenesis are important. Therefore, we assessed the effects of Amla treatment on antioxidant systems in $\mathrm{C} 2 \mathrm{C} 12$ myotubes. The activity of Nrf2, a key transcriptional factor involved in cellular antioxidant systems, was analyzed by luciferase reporter assay using a vector containing an ARE. As shown in Figure 3(a), Amla treatment dramatically increased ARE-driven luciferase activity. To further evaluate Nrf2 activation, we analyzed translocation of Nrf2 and observed that Amla treatment significantly increased Nrf2 translocation to the nucleus in $\mathrm{C} 2 \mathrm{C} 12$ myotubes (Figure 3(b)). Furthermore, we found that several antioxidant enzymes that are Nrf2 target genes, such as HO-1, NQO1, Cat, $\mathrm{Mn}-\mathrm{SOD}$, and $\mathrm{Cu}-\mathrm{SOD}$, were significantly upregulated at the transcriptional level following Amla treatment (Figure 3(c)).

3.5. Amla Treatment Exhibited Cytoprotective Effects on Cells Subjected to Oxidative Stress. To evaluate the cytoprotective effects of Amla treatment against oxidative stress, we first evaluated cell viability using the MTT assay in cells treated with t-BHP for $6 \mathrm{~h}$ to induce oxidative stress (Figure 4(a)). As shown in Figure $4(\mathrm{~b})$, t-BHP treatment $(250 \mu \mathrm{M}$ and $500 \mu \mathrm{M})$ induced cytotoxicity in a dose-dependent manner, whereas this was significantly attenuated by pretreatment with Amla for $48 \mathrm{~h}$. Next, to evaluate oxidative stress status, we analyzed ROS levels in $\mathrm{C} 2 \mathrm{C} 12$ myotubes treated with $\mathrm{t}-$ BHP for $2 \mathrm{~h}$ (Figure 4(a)). Without t-BHP treatment, Amla pretreatment significantly reduced ROS levels, whereas tBHP treatment increased ROS levels in a dose-dependent manner, which was significantly suppressed by pretreatment with Amla for $48 \mathrm{~h}$ (Figure 4(c)). We then evaluated oxygen consumption under oxidative stress conditions in $\mathrm{C} 2 \mathrm{C} 12$ myotubes with or without Amla pretreatment (Figure 4(a)). In control myotubes, t-BHP at high doses caused a timedependent increase in oxygen consumption, followed by a gradual decline, exhibiting a maximum OCR of $109.5 \pm 7.3 \%$ that of basal OCR. Amla pretreatment increased maximum OCR in the presence of $\mathrm{t}-\mathrm{BHP}(113.7 \pm 9.7 \%$ and $120.6 \pm 10.0 \%$ that of basal OCR at $250 \mu \mathrm{M}$ and $500 \mu \mathrm{M} \mathrm{t}$-BHP, resp.). OCRs 


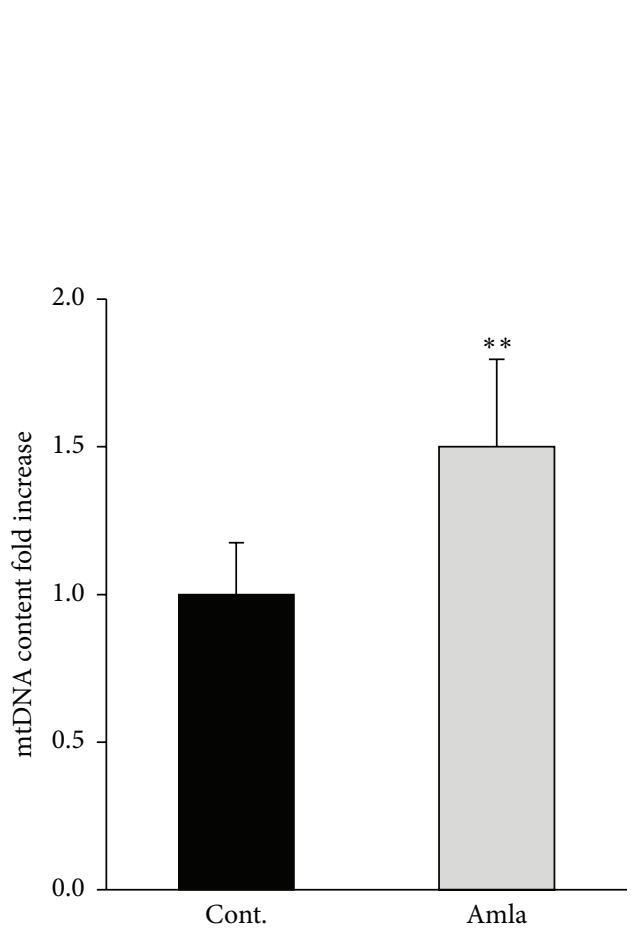

(a)

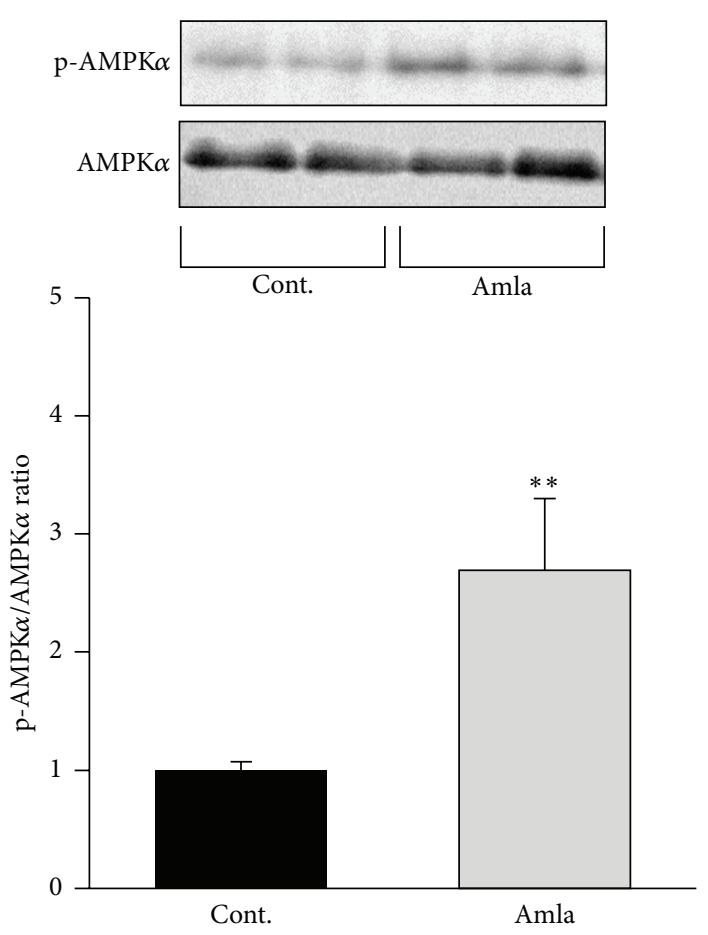

(b)

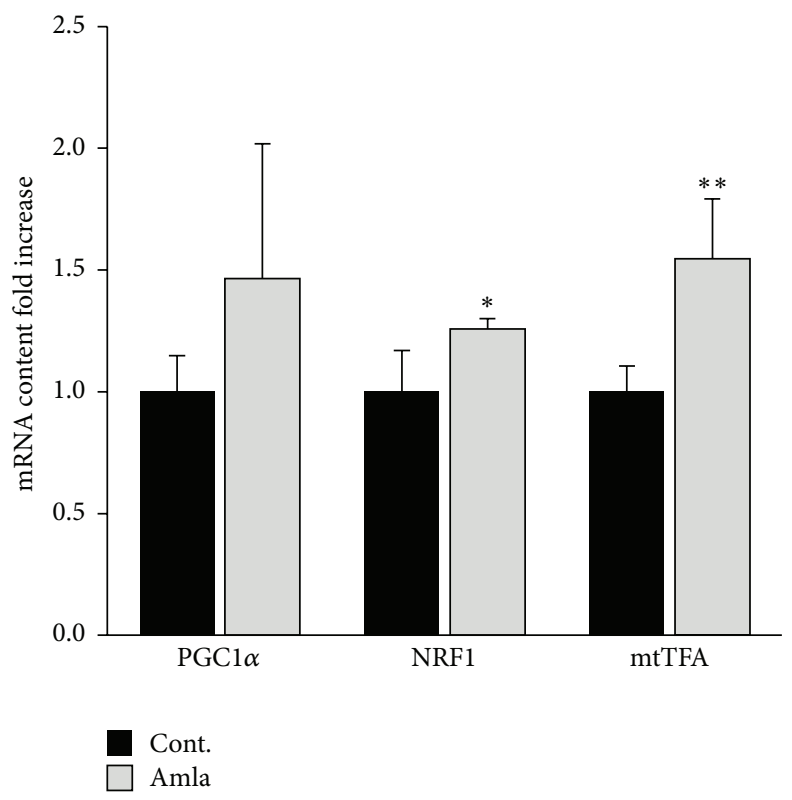

(c)

FIGURE 2: Amla treatment stimulated mitochondrial biogenesis by AMPK activation. C2C12 myotubes were incubated with Amla $(200 \mu \mathrm{g} / \mathrm{mL})$ for $48 \mathrm{~h}$. Cell lysates were prepared for western blotting, RT-qPCR, and mtDNA analysis. (a) Relative mtDNA content was determined by qPCR using specific primer sets for the mitochondrial and nuclear genome. ${ }^{* *} p<0.01 ; n=8$. (b) Phosphorylated AMPK $\alpha$ to total AMPK $\alpha$ ratios were determined by western blot. ${ }^{* *} p<0.01 ; n=6$. (c) Relative contents of PGC1 $\alpha$, NRF1, and mtTFA mRNAs were determined by RT-qPCR. ${ }^{*} p<0.05$ and ${ }^{* *} p<0.01 ; n=5$. $18 \mathrm{~S}$ rRNA was used as an internal control for RT-qPCR. 


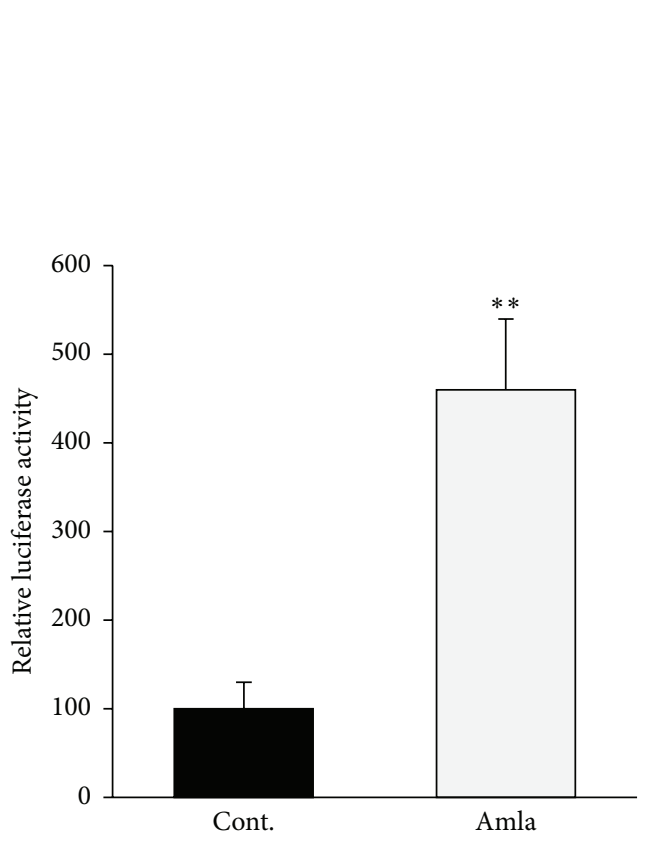

(a)

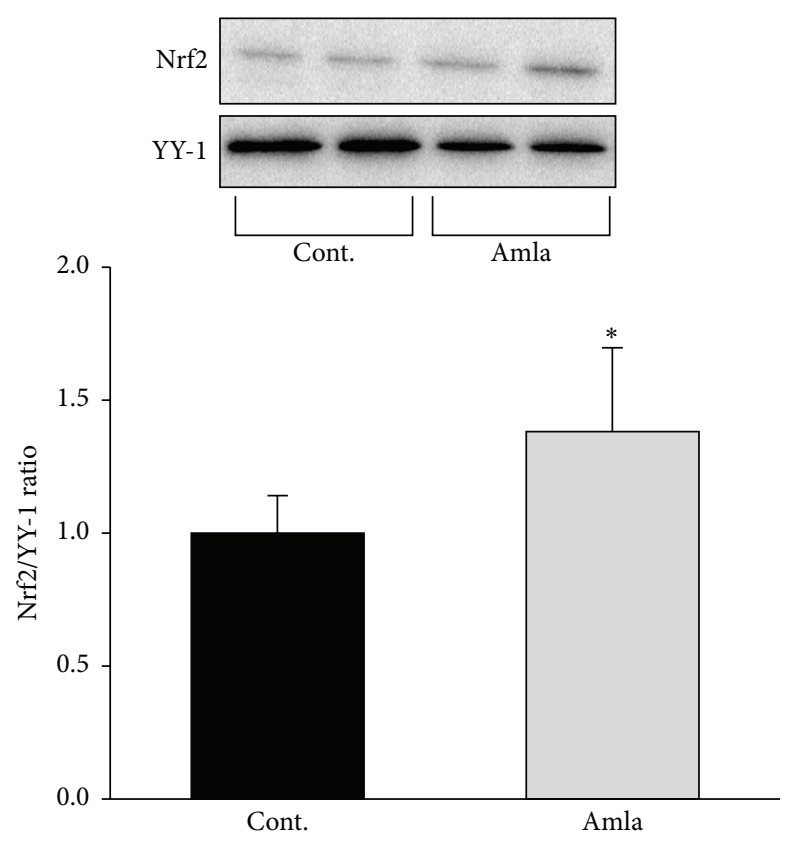

(b)

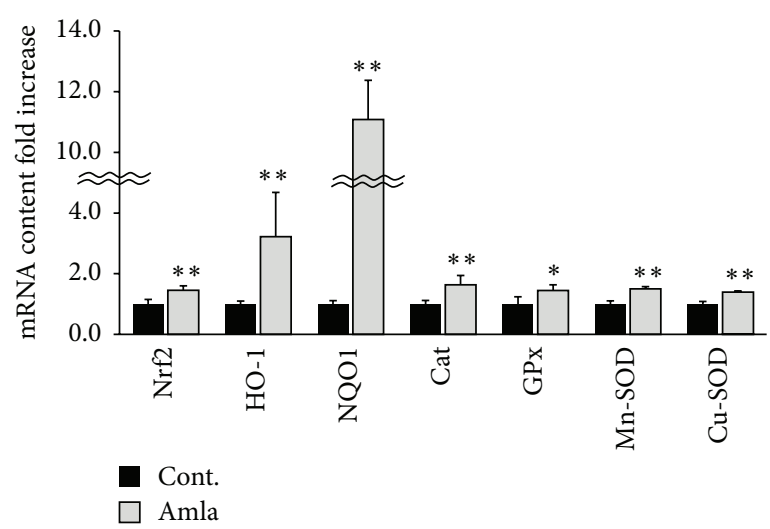

(c)

FIgURE 3: Amla treatment stimulated antioxidant systems by Nrf2 activation. (a) Activation of Nrf2 was analyzed using an ARE luciferase assay. Data are expressed as relative activities (reporter luciferase activity/control luciferase activity) as compared with data from control cells. ${ }^{* *} p<0.01 ; n=5$. (b and c) C2C12 myotubes were incubated with Amla $(200 \mu \mathrm{g} / \mathrm{mL})$ for $48 \mathrm{~h}$. (b) Nuclear lysates were analyzed by western blot, and YY-1 was used an internal control for nuclear protein. ${ }^{*} p<0.05 ; n=6$. (c) Relative levels of mRNA for antioxidant system related genes were analyzed by RT-qPCR. ${ }^{*} p<0.05 ;{ }^{* *} p<0.01 ; n=5.18 \mathrm{~S}$ rRNA was used as an internal control for RT-qPCR.

were unchanged following Amla pretreatment in the absence of t-BHP treatment (Figure $4(\mathrm{~d})$ ).

\section{Discussion}

In this study, we made three observations relevant to the beneficial effects of Amla treatment in murine skeletal muscle cells. First, Amla treatment stimulated mitochondrial function, specifically increasing mitochondrial spare respiratory capacity. Second, Amla treatment stimulated mitochondrial biogenesis and antioxidant systems along with activation of the AMPK and Nrf2 pathways. Third, Amla treatment protected cells against oxidative stress accompanied by increased oxygen consumption.
Amla treatment stimulated mitochondrial function by increasing mitochondrial spare respiratory capacity (Figure 1). There have been few studies reporting the ability of food ingredients to enhance mitochondrial spare respiratory capacity. In mouse cortical neuronal cultures, epicatechin and quercetin, both major polyphenols in plant-derived food materials, enhanced mitochondrial spare respiratory capacity and protected cells against oxygen-glucose deprivation stress [19]. Additionally, pharmaceutical approaches, such as administering thyroid hormones, can positively regulate mitochondrial spare respiratory capacity in skeletal muscle [20]. Decreased mitochondrial spare respiratory capacity was also reported in aging [21], and its depletion was implicated in various pathologies in high energy-requiring tissues, such 




(a)
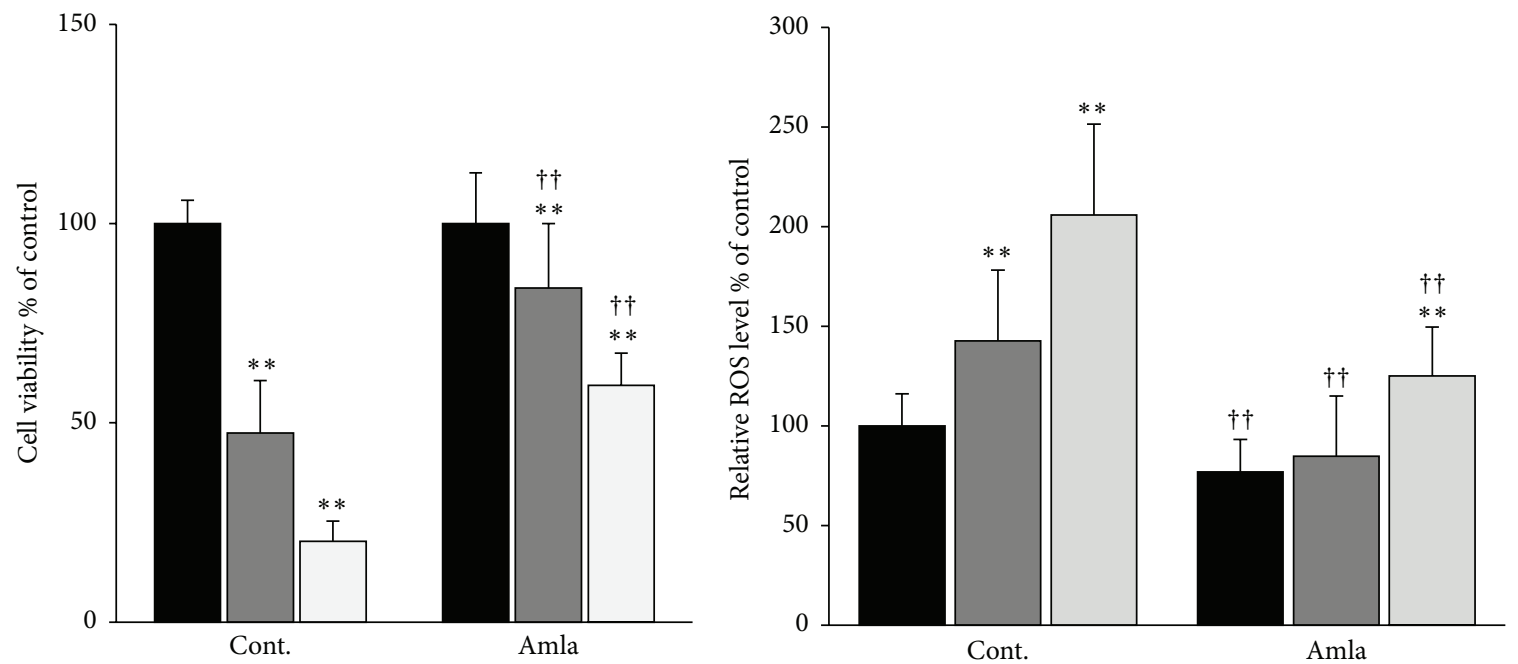

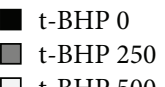

t-BHP 500

(b)

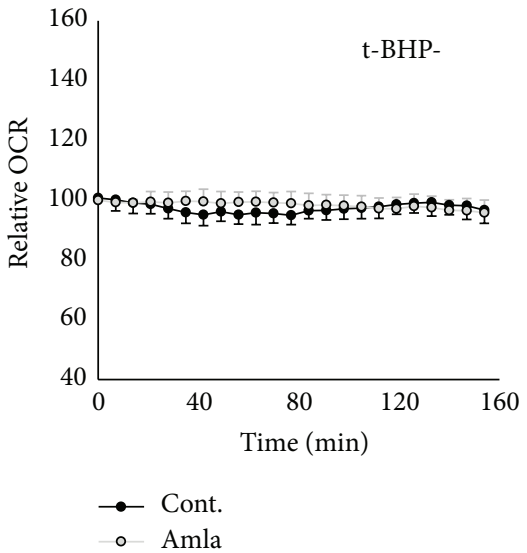

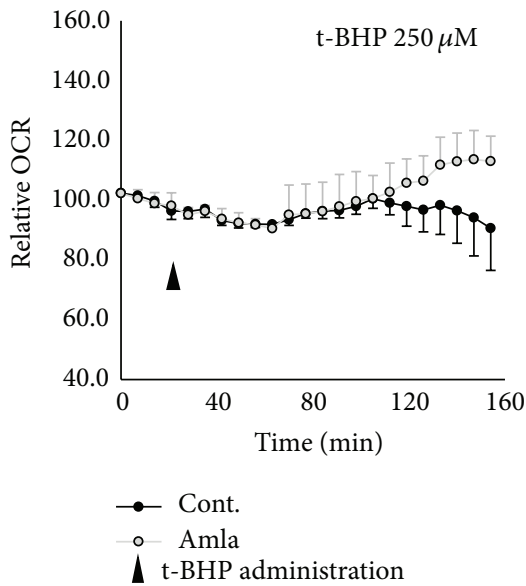

(d)

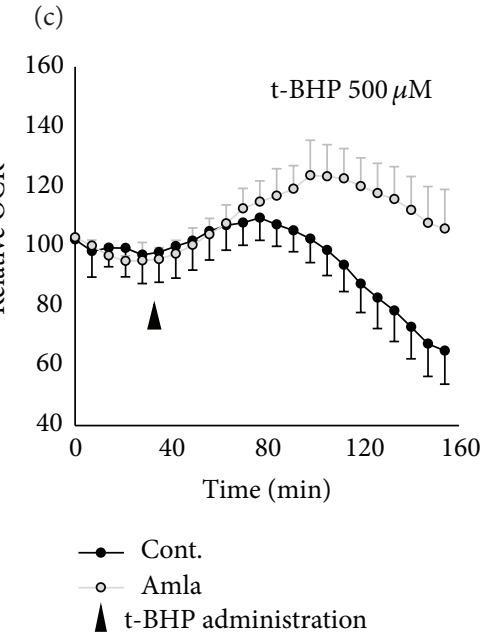

FIGURE 4: Amla treatment exhibited a cytoprotective effect against oxidative stress and concomitantly increased oxygen consumption. C2C12 myotubes were pretreated with Amla $(200 \mu \mathrm{g} / \mathrm{mL})$ for $48 \mathrm{~h}$ and then treated with t-BHP $(250 \mu \mathrm{M}$ or $500 \mu \mathrm{M})$. (a) Schematic showing the time points for three experiments performed to evaluate the cytoprotective effects of Amla treatment. (b) Cell viability was analyzed by MTT assay at $6 \mathrm{~h}$ after t-BHP treatment. ${ }^{* *} p<0.01$ versus t-BHP-untreated cells; ${ }^{\dagger \dagger} p<0.01$ versus Amla-untreated cells treated with each t-BHP concentration; $n=20$. (c) Relative ROS levels in cells were analyzed at $2 \mathrm{~h}$ after t-BHP stimulation. ${ }^{* *} p<0.01$ versus t-BHP-untreated cells; ${ }^{\dagger \dagger} p<0.01$ versus Amla-untreated cells treated with each t-BHP concentration; $n=12$. (d) OCR after t-BHP stimulation was analyzed following t-BHP injection after three basal OCR measurements. OCR was measured every $8 \mathrm{~min}$ for a total of $160 \mathrm{~min}$. Data are represented as relative-OCR values divided by the basal OCR values measured prior to t-BHP treatment $(n=10)$. 
as the heart, brain, and skeletal muscle [22-24]. Our findings suggested that Amla and other food ingredients are potentially novel approaches to improving age-related disorders in skeletal muscle by enhancing mitochondrial spare respiratory capacity. We confirmed that polyphenol rich Amla fractions prepared by adsorption chromatography stimulated mitochondrial respiratory spare capacity (see Supplemental Figures $1(\mathrm{~A})-1(\mathrm{C})$ in Supplementary Material available online at http://dx.doi.org/10.1155/2016/1735841). These results indicated that the polyphenols were the functional components responsible for the observed effects, and given that Amla was reported to contain higher polyphenol content [6], it was expected to be superior to other fruits in mitochondrial maintenance.

Amla treatment stimulated mitochondrial biogenesis and antioxidant systems along with activation of the AMPK $\alpha$ and Nrf2 pathways (Figures 2 and 3). Studies showed that both mitochondrial biogenesis and antioxidant systems could regulate mitochondrial spare respiratory capacity. Increased mitochondrial biogenesis and energy production caused by thyroid hormone treatment leads to stimulation of mitochondrial spare respiratory capacity in skeletal muscle [20]. In striatal neurons, decreased mitochondrial density accompanying mutant huntingtin expression indicated reduced mitochondrial spare respiratory capacity [25]. Furthermore, depletion of an antioxidant enzyme impaired mitochondrial spare respiratory capacity in cortical synaptosomes isolated from SOD2 null mice [26]. These reports were consistent with our findings, and we speculated that the effects of Amla treatment on mitochondrial spare respiratory capacity were related to activation of mitochondrial biogenesis and antioxidant systems. AMPK $\alpha$ and Nrf2 activation reportedly play key roles in mitochondrial biogenesis and antioxidant systems, and there are many studies showing that plantderived polyphenols activate AMPK $\alpha$ or Nrf2 $[27,28]$. In an animal model, dietary gallic acid protected mice from dietinduced obesity by stimulating the AMPK $\alpha /$ Sirt1/PGCl $\alpha$ pathway in liver, muscle, and brown adipose tissues [29]. Furthermore, ellagic acid consumption improved oxidantinduced endothelial dysfunction through Nrf2 activation [30]. These reports supported our findings, because the major polyphenols in Amla include gallic and ellagic acid [6]. Therefore, we proposed that gallic acid and ellagic acid were key factors involved in AMPK and Nrf2 activation following Amla treatment in our experiments.

Amla treatment protected cells against oxidative stress accompanied by increased oxygen consumption (Figure 4). Many reports showed that activation of antioxidant systems could increase cell survival in the presence of oxidative stress $[31,32]$. In our study, we observed that Amla treatment protected cells against t-BHP-induced cell death, likely by reducing ROS levels through activation of the Nrf2 pathway (Figures 4(b) and 4(c)). Various oxidative stresses led to increased oxygen consumption, suggesting a critical role for mitochondrial spare respiratory capacity in cell survival and maintenance of biological functions under oxidative stress $[15,33]$. We observed that t-BHP treatment increased OCR and that this was likely mediated by the spare respiratory capacity against oxidative stress. Additionally, pretreatment with Amla elevated the maximum OCR against t-BHP treatment (Figure 4(d)). Therefore, we speculated that not only activation of antioxidant systems but also increased mitochondrial function contributed to the cytoprotective effects of Amla treatment against oxidative stress.

In addition to being toxic, ROS are involved in various physiological processes as messenger molecules [34]. Signaling by insulin, several growth factors, and transcriptional factors can be mediated by physiological ROS levels [35, 36]. Because of these multiple roles, ROS homeostasis is regulated by numerous systems [37]. In our experiments, the magnitude by which Amla treatment decreased ROS increased with t-BHP concentration (Figure 4(c)). Therefore, our findings suggested that Amla treatment might remove excessive and damaging levels of ROS without neutralizing the ROS required for physiological function.

One limitation of our study was that it only demonstrated the effects of Amla treatment in vitro. Component analysis and previous research strongly suggested that polyphenols contributed to the effects of Amla treatment $[29,30]$ and that Amla contained various kinds of polyphenols, with gallic acid as a major component [6]. In one report, gallic acid was absorbed better in humans as compared to other polyphenols [38], and a previous clinical study investigating the bioavailability of gallic acid from red wine showed that glucuronidated and intact forms of gallic acid were detected in plasma [39]. These findings indicated that gallic acid in Amla may partially account for the biological relevance of our findings. Further experiments will be needed to clarify whether the effects of Amla treatment reported here can be reproduced in vivo.

Our results indicated that Amla treatment enhanced mitochondrial spare respiratory capacity, which was supported by its effect on mitochondrial biogenesis and antioxidant systems, through activation of the AMPK $\alpha$ and Nrf2 pathways, respectively. Furthermore, we found that Amla treatment decreased ROS levels and increased cell viability in the presence of t-BHP induced oxidative stress. We attributed the cytoprotective effects of Amla treatment not only to activation of antioxidant systems, but also to enhancement of the mitochondrial spare respiratory capacity (Figure 5).

\section{Conclusions}

In conclusion, our study demonstrated that Amla enhanced mitochondrial spare respiratory capacity through activation of mitochondrial biogenesis and antioxidant systems. Furthermore, we showed that these effects of Amla resulted in decreased ROS levels and increased cell viability under oxidative stress conditions. Therefore, our findings suggested novel potential mechanisms for the beneficial effects associated with Amla intake, including decreased oxidative stress.

\section{Competing Interests}

Hirotaka Yamamoto, Takao Ikami, Kohei Kiriyama, and Taishi Ishibashi are employees of Miki Corporation. Miki 


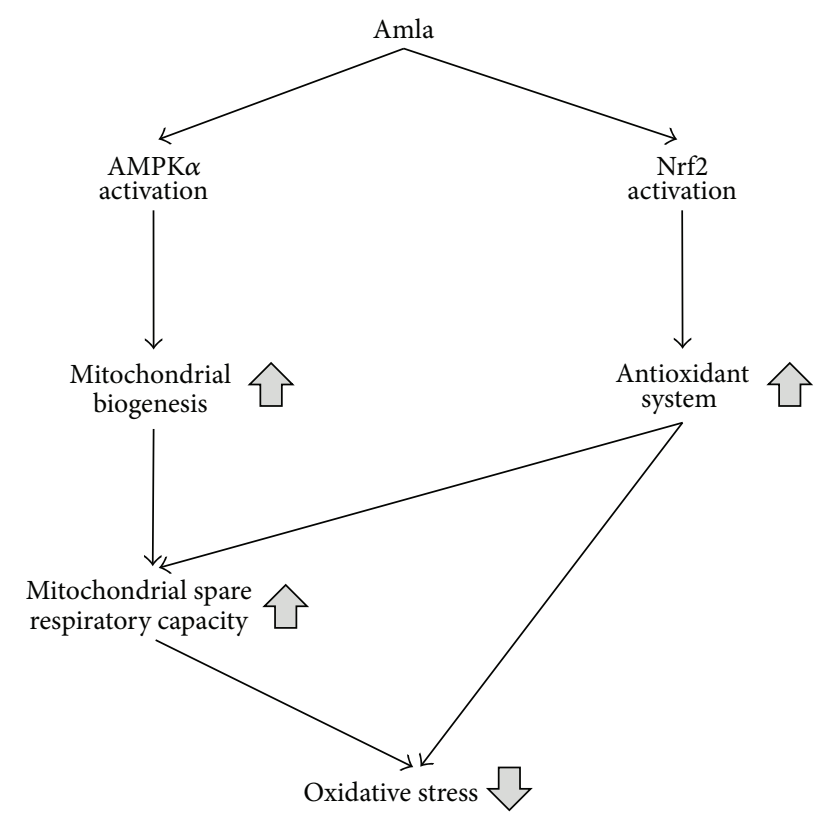

Figure 5: Schematic figure showing the effects of Amla treatment resulting in reduced oxidative stress.

Corporation has a food product containing Amla extract. This work was conducted in collaboration with Shiga University of Medical Science (SUMS). Hirotaka Yamamoto is a Visiting Assistant Professor at SUMS.

\section{Authors' Contributions}

Hirotaka Yamamoto and Katsutaro Morino conceived and designed the research; Hirotaka Yamamoto, Katsutaro Morino, Taishi Ishibashi, and Kohei Kiriyama performed experiments; Hirotaka Yamamoto and Katsutaro Morino analyzed data; Hirotaka Yamamoto, Katsutaro Morino, and Lemecha Mengistu interpreted the experimental results; Hirotaka Yamamoto and Katsutaro Morino prepared the figures and drafted the paper; Hirotaka Yamamoto, Katsutaro Morino, Takao Ikami, and Hiroshi Maegawa edited, revised, and approved the final version of paper.

\section{Acknowledgments}

The authors thank Misako Kitano, Takahiro Okuda, Yuichiro Mori, and Takashi Fujii for their technical assistance. The Department of Medicine, SUMS, received research promotion grants (Shougaku Kifukin) from Astellas, AstraZeneca, Bayer, Boehringer-Mannheim, Bristol-Myers Squibb, Chugai Pharma, Daiichi-Sankyo, Dainippon Sumitomo, Eisai, Eli Lilly, Fuji Yakuhin, Glaxo Smithkline, Kaken Pharmaceutical, Kaneka Medix, Kissei, Kowa Pharmaceuticals, Kyowa-hakko-Kirin, Miki Corporation, Mitsubishi Tanabe, Mochida, MSD, Nihon Medi-Physics, Nipro, Novartis, Novo Nordisk, Ono Pharmaceutical, Otsuka Pharmaceutical, Pfizer, Sanofi, Sanwa Kagaku Kenkyusho, Shionogi, Sunstar, Taisho-Toyama, Takeda, Teijin Pharma, Terumo, and Torii
Pharmaceutical. The research topics funded by these grants are unrestricted.

\section{References}

[1] S. S. Patel and R. K. Goyal, "Emblica officinalis Geart.: a comprehensive review on phytochemistry, pharmacology and ethnomedicinal uses," Research Journal of Medicinal Plant, vol. 6, no. 1, pp. 6-16, 2012.

[2] P. Bhandari and M. Kamdod, "Emblica officinalis (Amla): a review of potential therapeutic applications," International Journal of Green Pharmacy, vol. 6, no. 4, pp. 257-269, 2012.

[3] P. Usharani, N. Fatima, and N. Muralidhar, "Effects of Phyllanthus emblica extract on endothelial dysfunction and biomarkers of oxidative stress in patients with type 2 diabetes mellitus: a randomized, double-blind, controlled study," Diabetes, Metabolic Syndrome and Obesity: Targets and Therapy, vol. 6, pp. 275-284, 2013.

[4] A. Bhattacharya, A. Chatterjee, S. Ghosal, and S. K. Bhattacharya, "Antioxidant activity of active tannoid principles of Emblica officinalis (amla)," Indian Journal of Experimental Biology, vol. 37, no. 7, pp. 676-680, 1999.

[5] V. Damodara Reddy, P. Padmavathi, S. Gopi, M. Paramahamsa, and N. C. Varadacharyulu, "Protective effect of Emblica officinalis against alcohol-induced hepatic injury by ameliorating oxidative stress in rats," Indian Journal of Clinical Biochemistry, vol. 25, no. 4, pp. 419-424, 2010.

[6] T. Yokozawa, H. Y. Kim, L. R. Juneja et al., "Amla (Emblica officinalis Gaertn.) attenuates age-related renal dysfunction by oxidative stress," Journal of Agricultural and Food Chemistry, vol. 55, no. 19, pp. 7744-7752, 2007.

[7] T. Yokozawa, H. Y. Kim, H. J. Kim, T. Okubo, D.-C. Chu, and L. R. Juneja, "Amla (Emblica officinalis Gaertn.)prevents dyslipidaemia and oxidative stress in the ageing process," British Journal of Nutrition, vol. 97, no. 6, pp. 1187-1195, 2007.

[8] H. Y. Kim, T. Okubo, L. R. Juneja, and T. Yokozawa, "The protective role of amla (Emblica officinalis Gaertn.) against fructose-induced metabolic syndrome in a rat model," British Journal of Nutrition, vol. 103, no. 4, pp. 502-512, 2010.

[9] S. Ojha, M. Golechha, S. Kumari, and D. S. Arya, "Protective effect of Emblica officinalis (amla) on isoproterenol-induced cardiotoxicity in rats," Toxicology and Industrial Health, vol. 28, no. 5, pp. 399-411, 2012.

[10] S. S. Patel and R. K. Goyal, "Prevention of diabetes-induced myocardial dysfunction in rats using the juice of the Emblica officinalis fruit," Experimental and Clinical Cardiology, vol. 16, no. 3, pp. 87-91, 2011.

[11] M. S. Akhtar, A. Ramzan, A. Ali, and M. Ahmad, "Effect of amla fruit (Emblica officinalis Gaertn.) on blood glucose and lipid profile of normal subjects and type 2 diabetic patients," International Journal of Food Sciences and Nutrition, vol. 62, no. 6, pp. 609-616, 2011.

[12] A. Bratic and N.-G. Larsson, "The role of mitochondria in aging," The Journal of Clinical Investigation, vol. 123, no. 3, pp. 951-957, 2013.

[13] A. Safdar, M. J. Hamadeh, J. J. Kaczor, S. Raha, J. deBeer, and M. A. Tarnopolsky, "Aberrant mitochondrial homeostasis in the skeletal muscle of sedentary older adults," PLOS ONE, vol. 5, no. 5, Article ID e10778, 2010.

[14] C. Mancuso, G. Scapagini, D. Currò et al., "Mitochondrial dysfunction, free radical generation and cellular stress response 
in neurodegenerative disorders," Frontiers in Bioscience, vol. 12, pp. 1107-1123, 2007.

[15] B. G. Hill, B. P. Dranka, L. Zou, J. C. Chatham, and V. M. Darley-Usmar, "Importance of the bioenergetic reserve capacity in response to cardiomyocyte stress induced by 4hydroxynonenal," Biochemical Journal, vol. 424, no. 1, pp. 99107, 2009.

[16] D. B. Dennison, T. G. Brawley, and G. L. K. Hunter, "Rapid high-performance liquid chromatographic determination of ascorbic acid and combined ascorbic acid-dehydroascorbic acid in beverages," Journal of Agricultural and Food Chemistry, vol. 29, no. 5, pp. 927-929, 1981.

[17] S. Kim, J. M. Park, and C. H. Kim, "Ethanol production using whole plant biomass of Jerusalem artichoke by Kluyveromyces marxianus CBS1555," Applied Biochemistry and Biotechnology, vol. 169, no. 5, pp. 1531-1545, 2013.

[18] H. Yamamoto, K. Morino, Y. Nishio et al., "MicroRNA494 regulates mitochondrial biogenesis in skeletal muscle through mitochondrial transcription factor A and Forkhead box j3," American Journal of Physiology-Endocrinology and Metabolism, vol. 303, no. 12, pp. E1419-E1427, 2012.

[19] M. Nichols, J. Zhang, B. M. Polster et al., "Synergistic neuroprotection by epicatechin and quercetin: activation of convergent mitochondrial signaling pathways," Neuroscience, vol. 308, pp. 75-94, 2015.

[20] R. Lesmana, R. A. Sinha, B. K. Singh et al., "Thyroid hormone stimulation of autophagy is essential for mitochondrial biogenesis and activity in skeletal muscle," Endocrinology, vol. 157, no. 1, pp. 23-38, 2016.

[21] B. Rohrer, M. Bandyopadhyay, and C. Beeson, "Reduced metabolic capacity in aged primary retinal pigment epithelium (RPE) is correlated with increased susceptibility to oxidative stress," Advances in Experimental Medicine and Biology, vol. 854, pp. 793-798, 2016.

[22] C. Desler, T. L. Hansen, J. B. Frederiksen, M. L. Marcker, K. K. Singh, and L. Juel Rasmussen, "Is there a link between mitochondrial reserve respiratory capacity and aging?" Journal of Aging Research, vol. 2012, Article ID 192503, 9 pages, 2012.

[23] B. E. Sansbury, S. P. Jones, D. W. Riggs, V. M. Darley-Usmar, and B. G. Hill, "Bioenergetic function in cardiovascular cells: the importance of the reserve capacity and its biological regulation," Chemico-Biological Interactions, vol. 191, no. 1-3, pp. 288-295, 2011.

[24] N. Yadava and D. G. Nicholls, "Spare respiratory capacity rather than oxidative stress regulates glutamate excitotoxicity after partial respiratory inhibition of mitochondrial complex I with rotenone," The Journal of Neuroscience, vol. 27, no. 27, pp. 73107317, 2007.

[25] A. Siddiqui, S. R. Sánchez, M. D. R. Castro et al., "Mitochondrial DNA damage is associated with reduced mitochondrial bioenergetics in Huntington's disease," Free Radical Biology and Medicine, vol. 53, no. 7, pp. 1478-1488, 2012.

[26] J. M. Flynn, S. W. Choi, N. U. Day, A. A. Gerencser, A. Hubbard, and S. Melov, "Impaired spare respiratory capacity in cortical synaptosomes from Sod2 null mice," Free Radical Biology and Medicine, vol. 50, no. 7, pp. 866-873, 2011.

[27] M. Zang, S. Xu, K. A. Maitland-Toolan et al., "Polyphenols stimulate AMP-activated protein kinase, lower lipids, and inhibit accelerated atherosclerosis in diabetic LDL receptor-deficient mice," Diabetes, vol. 55, no. 8, pp. 2180-2191, 2006.

[28] H. Erlank, A. Elmann, R. Kohen, and J. Kanner, "Polyphenols activate $\mathrm{Nrf} 2$ in astrocytes via $\mathrm{H}_{2} \mathrm{O}_{2}$, semiquinones, and quinones," Free Radical Biology and Medicine, vol. 51, no. 12, pp. 2319-2327, 2011.

[29] K. V. Doan, C. M. Ko, A. W. Kinyua et al., "Gallic acid regulates body weight and glucose homeostasis through AMPK activation," Endocrinology, vol. 156, no. 1, pp. 157-168, 2015.

[30] Y. Ding, B. Zhang, K. Zhou et al., "Dietary ellagic acid improves oxidant-induced endothelial dysfunction and atherosclerosis: role of Nrf2 activation," International Journal of Cardiology, vol. 175, no. 3, pp. 508-514, 2014.

[31] R.-Z. Zhong, Y. Fang, G.-X. Qin, H.-Y. Li, and D.-W. Zhou, "Tea catechins protect goat skeletal muscle against $\mathrm{H}_{2} \mathrm{O}_{2}$-induced oxidative stress by modulating expression of phase 2 antioxidant enzymes," Journal of Agricultural and Food Chemistry, vol. 63, no. 36, pp. 7921-7928, 2015.

[32] J. S. Kang, M. H. Han, G.-Y. Kim et al., "Schisandrae semen essential oil attenuates oxidative stress-induced cell damage in $\mathrm{C} 2 \mathrm{C} 12$ murine skeletal muscle cells through Nrf2-mediated upregulation of HO-1," International Journal of Molecular Medicine, vol. 35, no. 2, pp. 453-459, 2015.

[33] B. P. Dranka, B. G. Hill, and V. M. Darley-Usmar, "Mitochondrial reserve capacity in endothelial cells: the impact of nitric oxide and reactive oxygen species," Free Radical Biology and Medicine, vol. 48, no. 7, pp. 905-914, 2010.

[34] H. Sies, "Role of metabolic $\mathrm{H}_{2} \mathrm{O}_{2}$ generation: redox signaling and oxidative stress," The Journal of Biological Chemistry, vol. 289, no. 13, pp. 8735-8741, 2014.

[35] D. Heffetz, L. Bushkin, R. Dror, and Y. Zick, "The insulinomimetic agents $\mathrm{H}_{2} \mathrm{O}_{2}$ and vanadate stimulate protein tyrosine phosphorylation in intact cells," The Journal of Biological Chemistry, vol. 265, no. 5, pp. 2896-2902, 1990.

[36] M. Sundaresan, Z.-X. Yu, V. J. Ferrans, K. Irani, and T. Finkel, "Requirement for generation of $\mathrm{H}_{2} \mathrm{O}_{2}$ for platelet-derived growth factor signal transduction," Science, vol. 270, no. 5234, pp. 296-299, 1995.

[37] A. Rahal, A. Kumar, V. Singh et al., "Oxidative stress, prooxidants, and antioxidants: the interplay," BioMed Research International, vol. 2014, Article ID 761264, 19 pages, 2014.

[38] M. Daglia, A. Di Lorenzo, S. F. Nabavi, Z. S. Talas, and S. M. Nabavi, "Polyphenols: well beyond the antioxidant capacity: gallic acid and related compounds as neuroprotective agents: you are what you eat!" Current Pharmaceutical Biotechnology, vol. 15, no. 4, pp. 362-372, 2014.

[39] E. Cartron, G. Fouret, M.-A. Carbonneau et al., "Red-wine beneficial long-term effect on lipids but not on antioxidant characteristics in plasma in a study comparing three types of wine-description of two $O$-methylated derivatives of gallic acid in humans," Free Radical Research, vol. 37, no. 9, pp. 1021$1035,2003$. 


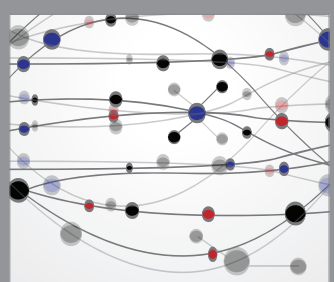

The Scientific World Journal
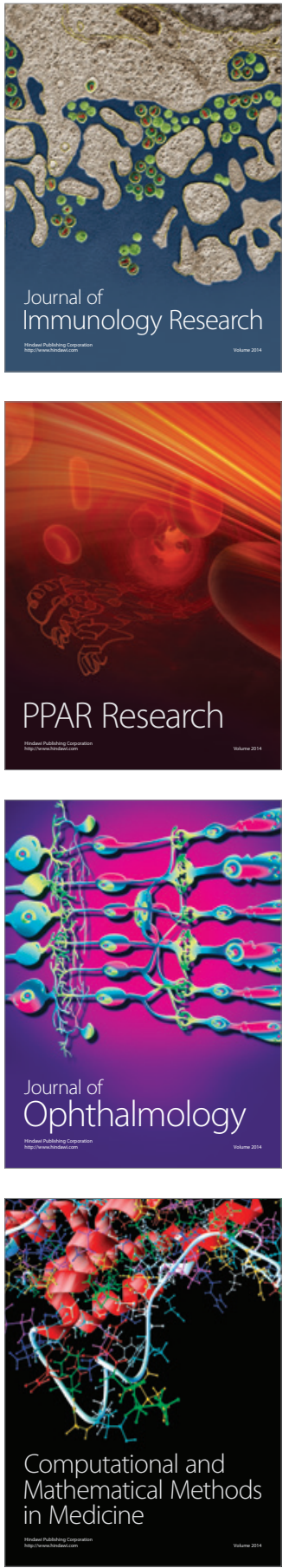

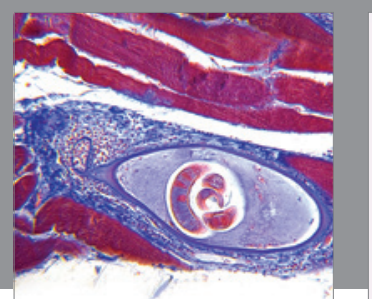

Gastroenterology Research and Practice



\section{Hindawi}

Submit your manuscripts at

http://www.hindawi.com
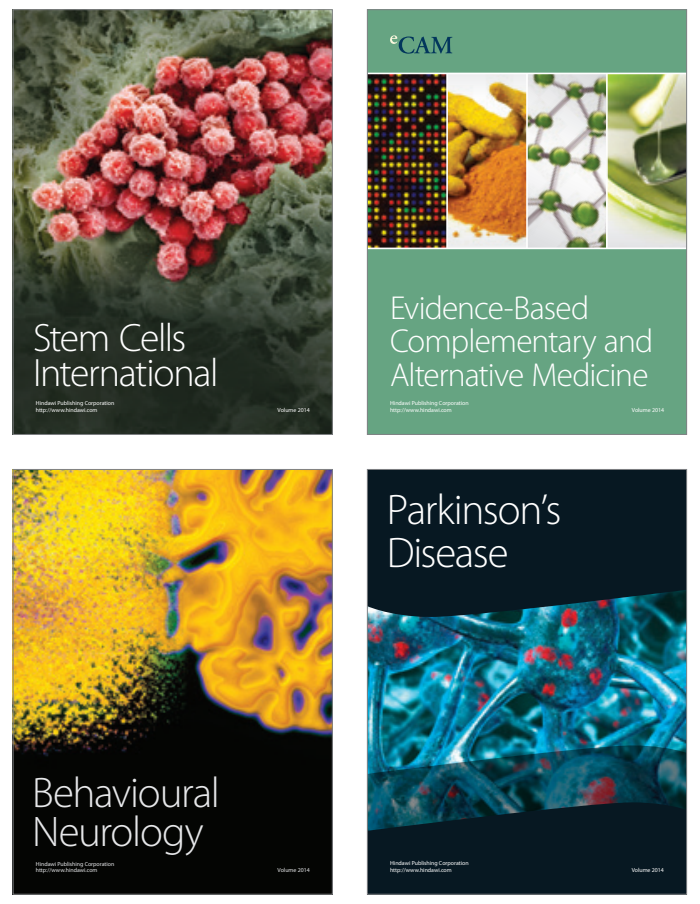
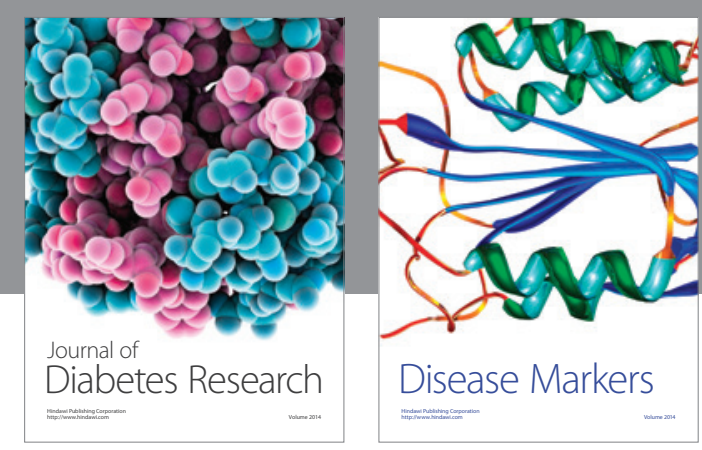

Disease Markers
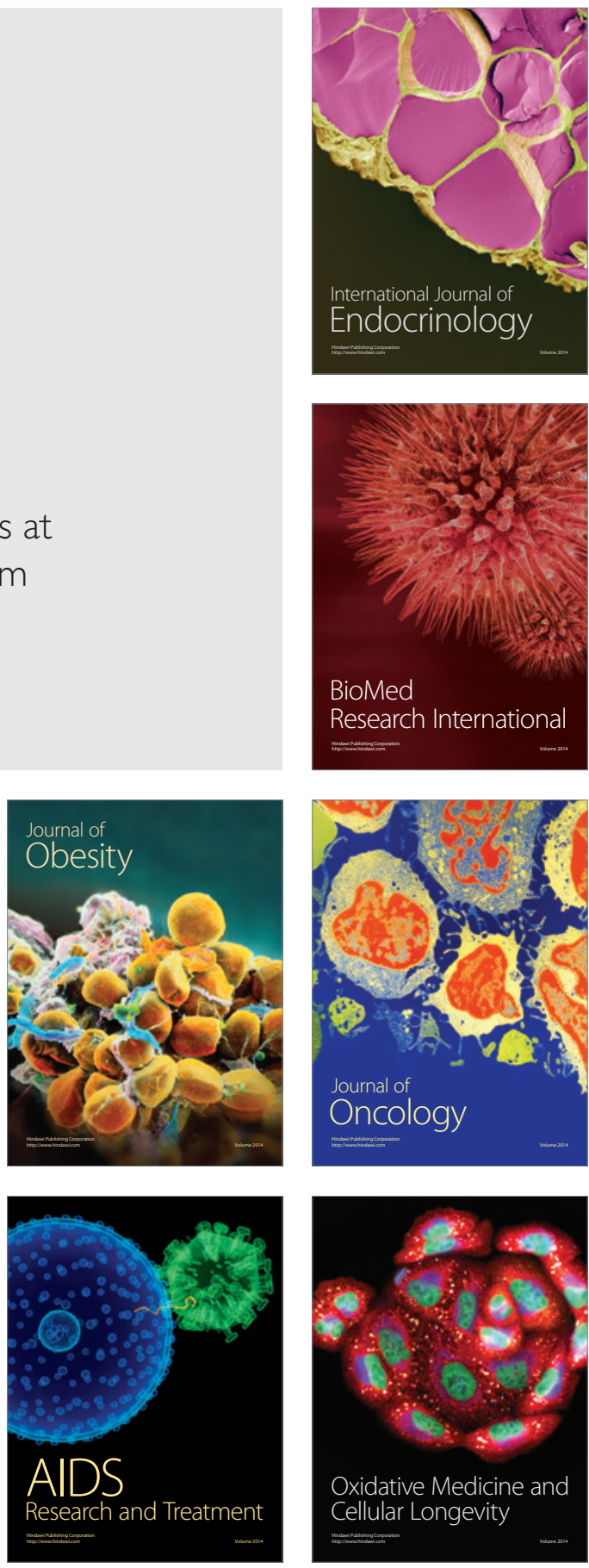